EPJ Web of Conferences 86, 00067 (2015)

DOI: $10.1051 /$ epjconf/ 20158600067

(C) Owned by the authors, published by EDP Sciences, 2015

\title{
GALS - setup for production and study of heavy neutron rich nuclei
}

\author{
Sergey Zemlyanoy ${ }^{1, a}$, Valery Zagrebaev ${ }^{1}$, Eduard Kozulin ${ }^{1}$, Yury Kudryavtsev ${ }^{2}$, Valentin Fedosseev ${ }^{3}$, Robert Bark ${ }^{4}$, \\ Zenon Janas ${ }^{5}$ and Hosam Othman ${ }^{6}$ \\ ${ }^{1}$ Joint Institute for Nuclear Research, 141980 Dubna, Russia \\ ${ }^{2}$ Instituut voor Kern-en Stralingsfysica, K.U. Leuven, 3001 Leuven, Belgium \\ ${ }^{3}$ CERN, 1211, Geneva 23, Switzerland \\ ${ }^{4}$ iThemba LABS, Nat. Research Foundation, Somerset West 7129, South Africa \\ ${ }^{5}$ Faculty of Physics, University of Warsaw, Hoza 69, PL-00681 Warsaw, Poland \\ ${ }^{6}$ Physics Department, Faculty of Science, Menoufiya University, Shibin El-kom, Menoufiya, Egypt
}

\begin{abstract}
The present limits of the upper part of the nuclear map are very close to stability while the unexplored area of heavy neutron-rich nuclides along the neutron closed shell $N=126$ below ${ }^{208} \mathrm{~Pb}$ is extremely important for nuclear astrophysics investigations and, in particular, for the understanding of the r-process of astrophysical nucleosynthesis. This area of the nuclear map can be reached neither in fusion-fission reactions nor in fragmentation processes widely used nowadays for the production of exotic nuclei. A new way was recently proposed for the production of these nuclei via low-energy multi-nucleon transfer reactions. The estimated yields of neutron-rich nuclei are found to be significantly high in such reactions and several tens of new nuclides can be produced, for example, in the near-barrier collision of ${ }^{136} \mathrm{Xe}$ with ${ }^{208} \mathrm{~Pb}$. A new setup is proposed to produce and study heavy neutron-rich nuclei located along the neutron closed shell $N=126$.
\end{abstract}

\section{Introduction}

A highlight in nuclear properties investigation of the last several years is a common effort of many collaborations to find an answer for the open questions in astrophysics. In this aspect, the unexplored area of heavy neutron rich nuclei is extremely important for the understanding of the $r$-process of astrophysical nucleogenesis. The $r$-process is responsible for the creation of about half of the heavy elements and its path critically depends on the neutron capture $Q$-value of very neutron-rich isotopes. Of special

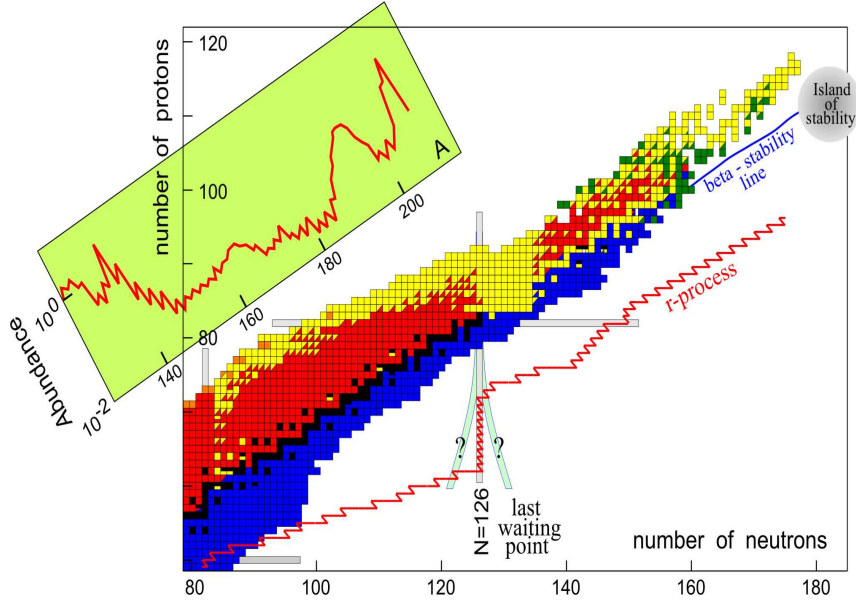

Figure 1. Upper part of the nuclear map. r-process of nucleosynthesis is shown schematically.

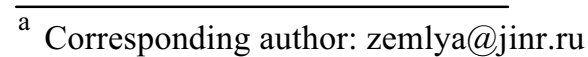

interest is the closed neutron shell $N=126$ which is the last "waiting point" in the $r$-process pathway. The halflives and other characteristics of these nuclei feed directly into the $r$-process scenario of supernovae explosions [1].

Let us note that the production of neutron rich nuclei heavier than $A>160$ encounters both physical and technical problems while medium mass neutron rich nuclei $(A \sim 100)$ are well accessible in fision reactions. Due to the increasing bending of the stability line to the neutron axis, fusion reactions of stable nuclei produce only proton rich isotopes of heavy elements. As a result, the number of neutron rich nuclei decreases with increasing $Z$, e.g. 19 neutron rich isotopes of cesium $(Z=$ $55)$ are known but only 4 of platinum $(Z=78)$. For elements heavier than fermium $(Z>100)$ only neutron deficient isotopes (located to the left of the stability line) have been synthesized so far. The present limits of the upper part of the nuclear map are very close to stability (Fig. 1). The area of heavy neutron-rich nuclides along the neutron closed shell $N=126$, extremely important for nuclear astrophysics investigations, remains unexplored so far.

Recently a new way was proposed for the production of these nuclei via low-energy multinucleon transfer reactions [2]. Their sufficiently high predicted yield opens a new opportunity in the studies at heavy-ion facilities and will have significant impact on future experiments. 


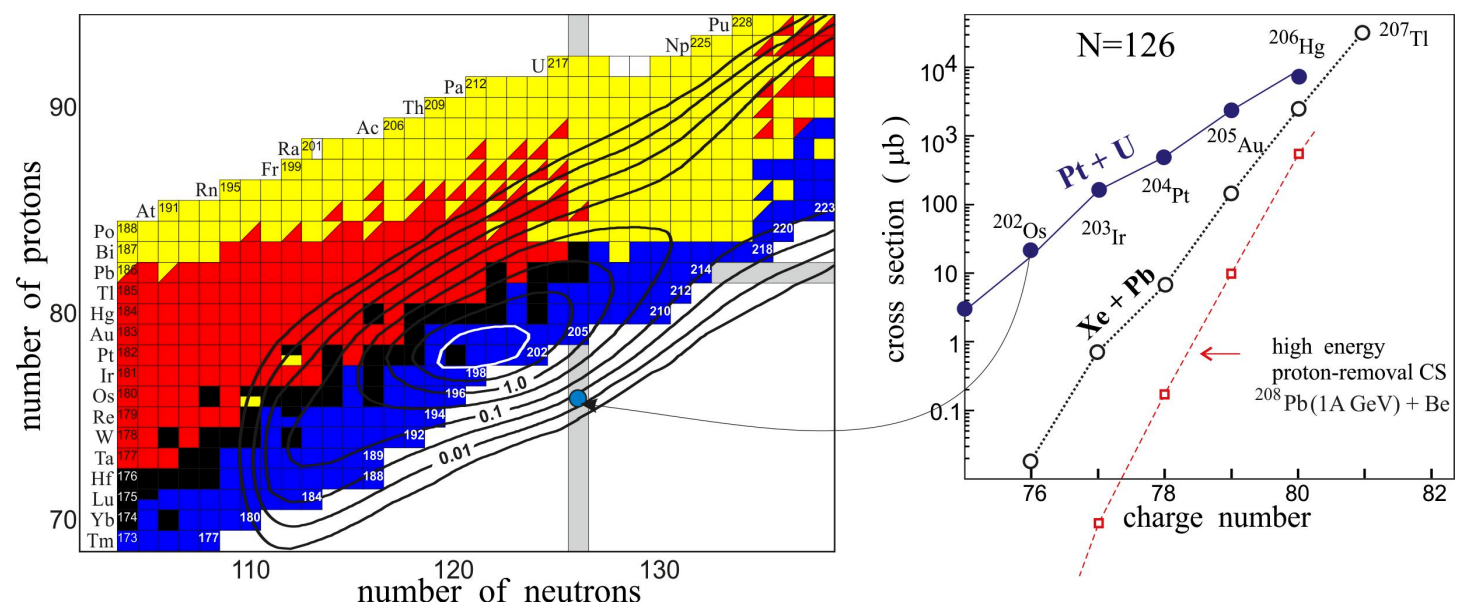

Figure 2. Cross sections for the formation of heavy nuclei in collisions of ${ }^{198} \mathrm{Pt}+{ }^{238} \mathrm{U}$ at center-of-mass energy of $700 \mathrm{MeV}$ [2]. Curves in the left panel are isocross-section lines, marked in mb.

The present paper motivates the necessity of a new setup for production of heavy neutron rich nuclei by multinucleon transfer reactions, describes the principle of the proposed setup and its mass-separator, gas-cell, ionguide and laser systems as well as their expected parameters.

\section{Physics objectives}

Heavy neutron rich nuclei can be produced in three different ways: multi-nucleon transfer reactions; fusion reactions with extremely neutron rich radioactive nuclei and rapid neutron capture processes. Proton removal reactions from high energy heavy projectiles like $U$ and $\mathrm{Pb}$ can also be used for the production of neutron rich nuclei in the lead region [3], but the reaction cross section drops drastically for more neutron rich products (see Fig.2). Due to the low intensity of radioactive beams and low neutron fluxes in existing nuclear reactors the second and third methods seem to be unrealizable. On the contrary, the low-energy multi-nucleon transfer reactions, as well as the quasi-fission processes [4] (which are similar) are quite practical. These processes are less studied as compared with well studied few nucleon transfer reactions (see for example review paper [5]). The main problem here is just identification (separation) of reaction products by $\mathrm{Z}$ and $\mathrm{A}$. Existing spectrometers (like PRISMA and VAMOS) cannot identify by $Z$ number heavy reaction product with $\mathrm{A}>70$.

Theoretical estimations show that multinucleon transfer reactions can be used for production of new neutron rich isotopes not only in the region of $Z \sim 80$ but also in the superheavy mass region. The cross-sections and production rates of such nuclei are calculated in [6] using a numerical solution of the coupled Langevin-type equations of motion. It is shown that several tens of new nuclides in the region of $N=126$ and $Z \sim 75$ can be produced, for example, in the near-barrier collision of ${ }^{136} \mathrm{Xe}$ with ${ }^{208} \mathrm{~Pb}$. Even higher cross sections have been predicted for the production of new neutron rich nuclei in collisions of ${ }^{198} \mathrm{Pt}$ beam with ${ }^{238} \mathrm{U}$ target (Fig. 2).
Neutron rich isotopes of transfermium elements $(Z>100)$ can also be produced in the multi-nucleon transfer reactions at low-energy collisions of actinide nuclei. Neutron rich fermium isotopes $(A>260)$ are extremely interesting for several reasons. Firstly no neutron rich isotope of fermium has been observed yet (see Fig. 1). Secondly, the so-called "fermium gap" (isotopes ${ }^{258-260} \mathrm{Fm}$ with short half-lives for spontaneous fission) impedes formation of nuclei with $Z>100$ by the weak neutron fluxes realized in existing nuclear reactors. In this context, it is extremely interesting to know what the first $\beta$-decaying fermium isotope is and how long is its half-life.

\section{Experimental setup}

During the last two decades a combined method of $Z$ and $A / Q$ separation has been intensively studied and developed based on stopping nuclei in gas and subsequent resonance laser ionization. This method, referred to as In-Gas Laser Ionization and Spectroscopy (IGLIS), is based on stopping of nuclear reaction products in a gas cell and subsequent selective resonance laser ionization [7 - 15]. Such technique allows extracting nuclei with a given atomic number, while a separation of the single-ionized isotopes by their mass number can be done rather easily by a magnetic field. Half-lives of heavy neutron rich nuclei, which we are interested in (as a rule, $\beta^{-}$-decaying), are much longer than the extraction time of ions from a gas cell. The schematic layout of the proposed facility for the study of new isotopes is shown in Fig. 3.

Neutron rich isotopes of heavy elements are produced in multi-nucleon transfer reactions with heavy ions accelerated up to 5-10 MeV/nucleon (depending on projectile-target combination). The target, a foil of about $300 \mu \mathrm{g} / \mathrm{cm}^{2}$ thickness (or larger), is placed at the window of the gas cell (or inside it). Nuclear reaction products recoiling out from the target as multi-charged ions are termalized and neutralized by collisions with highly-pure argon or helium atoms used as a buffer gas. Then the atoms of interest (with a given $Z$ number) are ionized by means of two or three-step resonance laser irradiation and

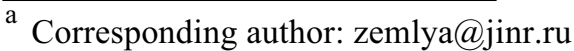






Figure 3. Schematic view of the proposed setup for resonance laser ionization of nuclear reaction products stopped and neutralized in gas and subsequently ionized, mass separated - and transported to detecting area.

are extracted by the gas flow through the exit into the vacuum chamber as singly charged ions $(Q=+1)$ with low energies of about $0.2 \mathrm{eV}$. Subsequently, the ions are confined in a radiofrequency ion guide system, which allows pumping out the residual buffer gas while transporting the ions towards the mass separator. Then the ions are accelerated up to $30-60 \mathrm{keV}$ and selected by the mass-separator. In this way a low-energy beam of singly-ionized ions with good optical quality (small emittance, energy spread less than $1 \mathrm{eV}$ ) is produced, allowing one to obtain a typical mass resolution of 1500 after dipole magnet. Atomic nuclei (after mass separation) have a definite charge number and a given (chosen) mass value; unwanted isobars and isotopes are significantly suppressed. This gives the possibility to perform subsequent high sensitive analysis of



Figure 4. The general view of the planned setup at cyclotron $\mathrm{U}-400 \mathrm{M}$ area. Several parts are already commissioned, e.g. laser set.

spectroscopic and decay properties of these nuclei, as well as a measurement of spins, magnetic dipole and

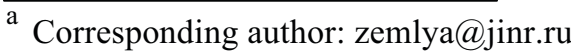

electric quadrupole moments and charge radii of these nuclei by means of laser spectroscopy.

\section{Conclusion and outlook}

The use of the designed setup for separation and study of heavy neutron rich nuclei does not require any specific and exotic beams of accelerated ions. The ion beams available at Join Institute for Nuclear Research (JINR) fully satisfy our requirements which look as follows: the ions - from ${ }^{16,18} \mathrm{O}$ to ${ }^{238} \mathrm{U}$ (i.e., quite different depending on the problem to be solved), beam energies $4.5-9$ $\mathrm{MeV} /$ nucleon (slightly above the Coulomb barrier), beam intensity up to $10^{13} \mathrm{pps}$ with a beam size at the target of $3-10 \mathrm{~mm}$ and beam emittance of $20 \mathbf{p} \cdot \mathrm{mm} \cdot \mathrm{mrad}$. The different heavy targets, including those of actinides, are expected to be used.

In-Gas Laser Ionization and Spectroscopy technique is proposed to be used for production and study of heavy neutron-rich nuclei. It is very suitable for the JINR cyclotron accelerators and the main direction of the JINR nuclear research. The method is rather universal and allows extracting besides heavy MNT reaction products also any other nuclei with half-lives longer than a few tens of milliseconds including neutron rich fission fragments, fusion reaction products and light exotic nuclei. Such studies are already performed at other setups, for example, in Finland [11] and Belgium [15], and also using a different approach as that of the hot cavity ion source at CERN [16]. Estimated efficiency of such setups is about $10 \%$ and depends on the half-life or the extracted ion. This is quite sufficient for the study of nuclear reaction products formed with cross sections of about 1 microbarn at a beam intensity of $0.1 \mathrm{p} \mu \mathrm{A}$. This method allows one to study the structure and decay properties of new exotic nuclei as well as spectroscopic properties of the corresponding atoms. At higher beam intensity this setup could be used also for separation and study of neutron rich long living superheavy nuclei 
produced in multi-nucleon transfer reactions with actinide targets (see above). This setup could definitely open a new opportunity in the studies at heavy-ion facilities and will have significant impact on future experiments.

\section{References}

1. S. Gupta, E.F. Brown, H. Schatz, P. Moeller, and K. L. Kratz, Astrophys. J. 662, 1188 (2007)

2. V. Zagrebaev, W. Greiner, Phys. Rev. Lett. 101 122701 (2008)

3. J. Kurcewicz et al., Phys. B 717, 371 (2012)

4. M.G. Itkis et al., Nucl. Phys. A 734, 136 (2004)

5. L. Corradi, G. Pollarolo, S. Szilner, J. of Phys. G 36, 113101 (2009)

6. V.I. Zagrebaev, Yu.Ts. Oganessian, M.G. Itkis, and Walter Greiner, Phys. Rev. C 73, 031602(R) (2006)

7. L. Vermeeren, N. Bijnens, M., Huyse, Y.A. Kudryavtsev, P. Van Duppen, J. Wauters, Z.N. Qamhieh, R.E. Silverans, P. Thoen, E. Vandeweert, Phys. Rev. Lett. 73, 1935 (1994)

8. Yu. Kudryavstev et al., Nucl. Instrum. Methods Phys. Res. B 114, 350 (1996).

9. Yu. Kudryavstev et al., Nucl. Instr. and Meth. Phys. Res. B 204, 336 (2003) .

10. M. Facina et al., Nuclear Instruments and Methods B 226, 401 (2004)

11. I.D. Moore et al., J. Phys. G, Nucl. Part. Phys. 31, S1499 (2005)

12. Y. Kudryavtsev et al., Nucl. Instrum. Methods Phys. Res. B 267, 2908 (2009)

13. R. Ferrer et al., NIM B 291, 29 (2012)

14. R. Ferrer et al., NIM B 317, 570 (2013).

15. Y. Kudryavtsev et al., NIM B 297, 7 (2013)

16. U.Köster et al., Spectrochim. Acta B 58, 1047 (2003) 